\title{
THE NON-SYMMETRIC WILSON POLYNOMIALS ARE THE BANNAI-ITO POLYNOMIALS
}

\author{
VINCENT X. GENEST, LUC VINET, AND ALEXEI ZHEDANOV
}

(Communicated by Mourad Ismail)

\begin{abstract}
The one-variable non-symmetric Wilson polynomials are shown to coincide with the Bannai-Ito polynomials. The isomorphism between the corresponding degenerate double affine Hecke algebra of type $\left(C_{1}^{\vee}, C_{1}\right)$ and the Bannai-Ito algebra is established. The Bannai-Ito polynomials are seen to satisfy an orthogonality relation with respect to a positive-definite and continuous measure on the real line. A non-compact form of the Bannai-Ito algebra is introduced and a four-parameter family of its infinite-dimensional and self-adjoint representations is exhibited.
\end{abstract}

\section{INTRODUCTION}

The purpose of this paper is fourfold: first, to show that the non-symmetric Wilson polynomials coincide with the Bannai-Ito polynomials; second, to identify the corresponding degenerate double affine Hecke algebra of type $\left(C_{1}^{\vee}, C_{1}\right)$ with the Bannai-Ito algebra; third, to exhibit a positive-definite, continuous orthogonality measure on the real line for the Bannai-Ito polynomials; and also, to present a non-compact form of the Bannai-Ito algebra and display a four-parameter family of its infinite-dimensional, self-adjoint representations.

We begin by reviewing the essentials of these two families of orthogonal functions and their associated algebraic structures.

1.1. The Bannai-Ito polynomials and algebra. The Bannai-Ito (BI) polynomials first appeared in the context of $P$ - and $Q-$ polynomial association schemes. In their book [3], E. Bannai and T. Ito identified the polynomials bearing their names as $q=-1$ analogs of the $q$-Racah polynomials in their classification of the orthogonal polynomials satisfying the Leonard duality [18]; see [2] for an overview.

The (monic) Bannai-Ito polynomials $\mathbf{B}_{n}(x)$ of degree $n$ in $x$, which depend on four parameters $\mathfrak{a}, \mathfrak{b}, \mathfrak{c}, \mathfrak{d}$, are defined by the three-term recurrence relation 1

$$
x \mathbf{B}_{n}(x)=\mathbf{B}_{n+1}(x)+\left(2 \mathfrak{a}+1-A_{n}-C_{n}\right) \mathbf{B}_{n}(x)+A_{n-1} C_{n} \mathbf{B}_{n-1}(x),
$$

Received by the editors July 10, 2015 and, in revised form, February 8, 2016.

2010 Mathematics Subject Classification. Primary 33C80, $20 \mathrm{C} 08$.

This work was presented on January 9, 2016 by the first author at the Joint Mathematics Meetings in the AMS Special Session on Special Functions and $q$-Series.

${ }^{1}$ With respect to the usual parametrization (e.g [23]) where the BI polynomials are denoted by $B_{n}\left(y ; \rho_{1}, \rho_{2}, r_{1}, r_{2}\right)$, we have taken $y=x / 2-1 / 4, \rho_{1}=\mathfrak{a}+1 / 4, \rho_{2}=\mathfrak{b}+1 / 4, r_{1}=-\mathfrak{c}-1 / 4$, $r_{2}=-\mathfrak{d}-1 / 4$. 
with $\mathbf{B}_{-1}(x)=0, \mathbf{B}_{0}(x)=1$ and where the coefficients read

$$
\begin{aligned}
& A_{n}= \begin{cases}\frac{(n+2 \mathfrak{a}+2 \mathfrak{c}+2)(n+2 \mathfrak{a}+2 \mathfrak{d}+2)}{2(n+\mathfrak{a}+\mathfrak{b}+\mathfrak{c}+\mathfrak{d}+2)} & n \text { even, } \\
\frac{(n+2 \mathfrak{a}+2 \mathfrak{b}+2)(n+2 \mathfrak{a}+2 \mathfrak{b}+2 \mathfrak{c}+2 \mathfrak{d}+3)}{2(n+\mathfrak{a}+\mathfrak{b}+\mathfrak{c}+\mathfrak{d}+2)} & n \text { odd },\end{cases} \\
& C_{n}= \begin{cases}-\frac{n(n+2 \mathfrak{c}+2 \mathfrak{d}+1)}{2(n+\mathfrak{a}+\mathfrak{b}+\mathfrak{c}+\mathfrak{d}+1)} & n \text { even, } \\
-\frac{(n+2 \mathfrak{b}+2 \mathfrak{c}+1)(n+2 \mathfrak{b}+2 \mathfrak{d}+1)}{2(n+\mathfrak{a}+\mathfrak{b}+\mathfrak{c}+\mathfrak{d}+1)} & n \text { odd. }\end{cases}
\end{aligned}
$$

The polynomials $\mathbf{B}_{n}(x)$ are eigenfunctions of the most general, self-adjoint, firstorder Dunkl shift operator preserving the space of polynomials of a given degree [23. Specifically, they satisfy the eigenvalue equation

$$
\mathcal{L} \mathbf{B}_{n}(x)=\lambda_{n} \mathbf{B}_{n}(x), \quad \lambda_{n}=(-1)^{n}(n+\mathfrak{a}+\mathfrak{b}+\mathfrak{c}+\mathfrak{d}+3 / 2),
$$

where $\mathcal{L}$ is the difference operator given by

$$
\begin{aligned}
\mathcal{L}= & \left(\frac{(x+2 \mathfrak{c}+1)(x+2 \mathfrak{d}+1)}{2 x+1}\right)\left(T_{x}^{+} R_{x}-\mathbb{1}\right) \\
& -\left(\frac{(x-2 \mathfrak{a}-1)(x-2 \mathfrak{b}-1)}{2 x-1}\right)\left(T_{x}^{-} R_{x}-\mathbb{1}\right)+(\mathfrak{a}+\mathfrak{b}+\mathfrak{c}+\mathfrak{d}+3 / 2) \mathbb{1},
\end{aligned}
$$

where $R_{x} f(x)=f(-x)$ is the reflection operator and where $T_{x}^{ \pm} f(x)=f(x \pm 1)$ are the discrete shift operators. The eigenvalue equation (1.2), together with the condition that the polynomials $\mathbf{B}_{n}(x)$ are monic (i.e. $\mathbf{B}_{n}(x)=x^{n}+\mathcal{O}\left(x^{n-1}\right)$ ), completely determine $\mathbf{B}_{n}(x)$. The bispectrality property of $\mathbf{B}_{n}(x)$, expressed through the relations (1.1) and (1.2), is encoded in an algebraic structure that has been called the Bannai-Ito algebra [23]; this associative algebra is obtained as follows. Let $K_{1}, K_{2}$ be the generators defined by

$$
K_{1}=\mathcal{L}, \quad K_{2}=X
$$

where $X$ is the "multiplication by $x$ " operator. Writing the anticommutator as $\{A, B\}=A B+B A$, one has 23 .

$$
\left\{K_{1}, K_{2}\right\}=K_{3}+\omega_{3}, \quad\left\{K_{2}, K_{3}\right\}=K_{1}+\omega_{1}, \quad\left\{K_{3}, K_{1}\right\}=K_{2}+\omega_{2},
$$

where the first relation of (1.5) is taken to define $K_{3}$. The structure constants $\omega_{i}$ have the expressions

$$
\begin{aligned}
& \omega_{1}=4(\mathfrak{a} \mathfrak{b}+\mathfrak{c} \mathfrak{d})+(\mathfrak{a}+\mathfrak{b}+\mathfrak{c}+\mathfrak{d})+1 / 2, \\
& \omega_{2}=2\left(\mathfrak{a}^{2}+\mathfrak{b}^{2}-\mathfrak{c}^{2}-\mathfrak{d}^{2}\right)+(\mathfrak{a}+\mathfrak{b}-\mathfrak{c}-\mathfrak{d}), \\
& \omega_{3}=4(\mathfrak{a} \mathfrak{b}-\mathfrak{c} \mathfrak{d})+(\mathfrak{a}+\mathfrak{b}-\mathfrak{c}-\mathfrak{d})
\end{aligned}
$$

The relations (1.5) define the abstract Bannai-Ito algebra. Note that the arbitrary structure constants can be viewed as being associated to a central element. This algebra has a Casimir operator

$$
Q=K_{1}^{2}+K_{2}^{2}+K_{3}^{2}
$$

which commutes with all generators $K_{i}, i=1,2,3$. In the realization (1.4), the Casimir operator takes the value

$$
Q=2\left(\mathfrak{a}^{2}+\mathfrak{b}^{2}+\mathfrak{c}^{2}+\mathfrak{d}^{2}\right)+(\mathfrak{a}+\mathfrak{b}+\mathfrak{c}+\mathfrak{d})+1 / 4 .
$$


When the parameters $\mathfrak{a}, \mathfrak{b}, \mathfrak{c}, \mathfrak{d}$ are real, the Bannai-Ito polynomials can only obey a finite orthogonality relation with respect to a discrete, positive measure; see [6] for details. This is related to the fact that for real values of the parameters, the selfadjoint representations of the Bannai-Ito algebra with spectrum (1.2), i.e. those that satisfy the $*$-relations $K_{i}^{\dagger}=K_{i}$ for $i=1,2,3$, can only be finite dimensional. Let us note here that the Bannai-Ito algebra has recently appeared as symmetry algebra for the Laplace-Dunkl [8] and Dirac-Dunkl [4] equations on the 2-sphere as well as in the Racah problem of $\mathfrak{o s p}(1 \mid 2)$ [7. Let us also mention that the Bannai-Ito polynomials sit at the top of the emerging "-1" scheme of orthogonal polynomials, which extends the Askey tableau [6, 13, 14, 23, 24, 29,

The Bannai-Ito algebra can be thought of as a $q=-1$ analog of the AskeyWilson algebra [30, which describes the deeper symmetries of the Askey-Wilson polynomials. In two papers [16, 17, Koornwinder established the relationship between the Askey-Wilson algebra and the double affine Hecke algebra associated with the rank-one root system of type $\left(C_{1}^{\vee}, C_{1}\right)$. These results relate to the large body of work on multivariate orthogonal polynomials and generalized CalogeroMoser systems associated with Cherednik algebras and root systems; see for example [15, 19, 25, 28]. In the present paper, we shall put the Bannai-Ito algebra and polynomials in a similar framework by exhibiting their relationship with the degenerate double affine Hecke algebra associated to the rank-one root system of type $\left(C_{1}^{\vee}, C_{1}\right)$ and with the non-symmetric Wilson polynomials introduced by Groenevelt in [10,11].

1.2. The one-variable non-symmetric Wilson polynomials and a rankone, degenerate double affine Hecke algebra. Let $t_{0}, t_{1}$ and $u_{0}, u_{1}$ be complex parameters and let $T_{0}, T_{1}$ be defined by

$$
\begin{aligned}
& T_{0}=\frac{\left(t_{0}+u_{0}-z+1 / 2\right)\left(t_{0}-u_{0}-z+1 / 2\right)}{1-2 z}\left(T_{z}^{-} R_{z}-\mathbb{1}\right)+t_{0} \mathbb{1}, \\
& T_{1}=\frac{\left(t_{1}+u_{1}+z\right)\left(t_{1}-u_{1}+z\right)}{2 z}\left(R_{z}-\mathbb{1}\right)+t_{1} \mathbb{1} .
\end{aligned}
$$

It is easy to check that $T_{i}^{2}=t_{i}^{2}$ for $i=0,1$; hence $T_{0}$ and $T_{1}$ are involutions. The non-symmetric Wilson polynomials $p_{n}(z)$ of degree $n$ in $z$, which depend on four parameters $t_{0}, t_{1}, u_{0}, u_{1}$, are defined as the unique (monic) polynomials satisfying the eigenvalue equation [10]

$$
\left(T_{0}+T_{1}\right) p_{n}(z)=\gamma_{n} p_{n}(z),
$$

where the eigenvalues $\gamma_{n}$ are given by

$$
\gamma_{n}= \begin{cases}t_{0}+t_{1}+m & n=2 m, \\ -\left(t_{0}+t_{1}+m\right) & n=2 m-1 .\end{cases}
$$

The polynomials $p_{n}(z)$ satisfy the complex orthogonality relation 10.

$$
\frac{1}{2 \pi i} \int_{\mathcal{C}} p_{n}(z) p_{m}(z) \Delta(z) \mathrm{d} z=h_{n} \delta_{n m},
$$

with respect to the weight function

$$
\begin{gathered}
\Delta(z)=\frac{\Gamma\left(t_{1}+u_{1}+z\right) \Gamma\left(t_{1}+u_{1}+1-z\right) \Gamma\left(t_{1}-u_{1}+z\right) \Gamma\left(t_{1}-u_{1}+1-z\right)}{\Gamma(2 z) \Gamma(1-2 z)} \\
\times \Gamma\left(t_{0}+u_{0}+1 / 2+z\right) \Gamma\left(t_{0}+u_{0}+1 / 2-z\right) \Gamma\left(t_{0}-u_{0}+1 / 2+z\right) \Gamma\left(t_{0}-u_{0}+1 / 2-z\right),
\end{gathered}
$$


where $h_{n} \neq 0$ and where $\mathcal{C}$ is the usual contour that runs along the imaginary axis and that is indented, if necessary, to separate the increasing sequence of poles from the decreasing sequence of poles in the weight function; see for example [1]. Sufficient conditions on the parameters $t_{i}, u_{i}$ for this contour to exist are easily found. The algebraic structure associated with the non-symmetric Wilson polynomials is as follows. Introduce the operators $U_{0}, U_{1}$ defined as 10 .

$$
U_{0}=-T_{0}+Z-1 / 2, \quad U_{1}=-T_{1}-Z,
$$

where $Z$ is the "multiplication by $z$ " operator. The degenerate double affine Hecke algebra associated to the non-symmetric Wilson polynomials has generators $T_{0}, T_{1}$, $U_{0}, U_{1}$ and relations

$$
\begin{gathered}
T_{0}^{2}=t_{0}^{2}, \quad T_{1}^{2}=t_{1}^{2}, \quad U_{0}^{2}=u_{0}^{2}, \quad U_{1}^{2}=u_{1}^{2}, \\
T_{0}+T_{1}+U_{0}+U_{1}=-\frac{1}{2} .
\end{gathered}
$$

These relations are easily verified in the realization (1.8), (1.12). This algebra can be viewed as a $q=1$ analog of the double affine Hecke algebra of type $\left(C_{1}^{\vee}, C_{1}\right)$ [20, 21]. The algebra (1.13) was also considered in [5]. In the following, we shall use an elementary generalization (1.13) for which $t_{i}, u_{i}$ for $i=0,1$ are considered as central elements rather than complex parameters. Following [22], this could be called the "universal" degenerate double affine Hecke algebra of type $\left(C_{1}^{\vee}, C_{1}\right)$.

1.3. Outline. The outline for the rest of the paper is straightforward. In Section 2, the non-symmetric Wilson polynomials are shown to coincide with the BannaiIto polynomials by comparison of their respective eigenvalue equations. The isomorphism between the (universal) degenerate double affine Hecke algebra and the Bannai-Ito algebra is given explicitly on the generators. In Section 3, we exhibit a positive-definite continuous orthogonality measure for the BI polynomials. We also display a non-compact form of the BI algebra and specify a four-parameter family of irreducible self-adjoint representations. A conclusion follows.

\section{BANNAI-ITO VS. NON-SYMMETRIC WILSON POLYNOMIALS}

In this section, the equivalence between the Bannai-Ito polynomials and the non-symmetric Wilson polynomials is demonstrated and the isomorphism between the Bannai-Ito algebra and the (universal) degenerate double affine Hecke algebra is established.

Proposition 1. Let $p_{n}\left(z ; t_{0}, t_{1}, u_{0}, u_{1}\right)$ be the monic non-symmetric Wilson polynomials defined by the eigenvalue equation (1.9) and let $\mathbf{B}_{n}(x ; \mathfrak{a}, \mathfrak{b}, \mathfrak{c}, \mathfrak{d})$ be the monic Bannai-Ito polynomials defined by (1.2). One has

$$
(-2)^{n} p_{n}\left(-\frac{x}{2}+\frac{1}{4} ; \frac{\mathfrak{c}+\mathfrak{d}}{2}+\frac{1}{4}, \frac{\mathfrak{a}+\mathfrak{b}}{2}+\frac{1}{4}, \frac{\mathfrak{c}-\mathfrak{d}}{2}, \frac{\mathfrak{a}-\mathfrak{b}}{2}\right)=\mathbf{B}_{n}(x ; \mathfrak{a}, \mathfrak{b}, \mathfrak{c}, \mathfrak{d}) .
$$

Hence the non-symmetric Wilson polynomials coincide with the Bannai-Ito polynomials, up to an affine transformation.

Proof. As $p_{n}\left(z ; t_{0}, t_{1}, u_{0}, u_{1}\right)$ and $\mathbf{B}_{n}(x ; \mathfrak{a}, \mathfrak{b}, \mathfrak{c}, \mathfrak{d})$ are determined uniquely by their eigenvalue equations (1.9) and (1.2) and the monicity condition, it suffices to show 
that under the affine transformation and reparametrization

$$
z \rightarrow-\frac{x}{2}+\frac{1}{4}, \quad\left(t_{0}, t_{1}, u_{0}, u_{1}\right) \rightarrow\left(\frac{\mathfrak{c}+\mathfrak{d}}{2}+\frac{1}{4}, \frac{\mathfrak{a}+\mathfrak{b}}{2}+\frac{1}{4}, \frac{\mathfrak{c}-\mathfrak{d}}{2}, \frac{\mathfrak{a}-\mathfrak{b}}{2}\right),
$$

the eigenvalue equation (1.9) gives (1.2). Under the above affine transformation, it is easily checked that one has

$$
T_{z}^{-} R_{z} \rightarrow T_{x}^{+} R_{x}, \quad R_{z} \rightarrow T_{x}^{-} R_{x}
$$

and hence that (1.9) becomes

$$
\begin{aligned}
& {\left[\frac{(x+2 \mathfrak{c}+1)(x+2 \mathfrak{d}+1)}{2 x+1}\left(T_{x}^{+} R_{x}-\mathbb{1}\right)+(\mathfrak{a}+\mathfrak{b}+\mathfrak{c}+\mathfrak{d}+1) \mathbb{1}\right.} \\
& \left.+\frac{(x-2 \mathfrak{a}-1)(x-2 \mathfrak{b}-1)}{2 x+1}\left(T_{x}^{-} R_{x}-\mathbb{1}\right)\right] p_{n}\left(-\frac{x}{2}+\frac{1}{4}\right)=2 \gamma_{n} p_{n}\left(-\frac{x}{2}+\frac{1}{4}\right) .
\end{aligned}
$$

Upon adding $(1 / 2) \mathbb{1}$ on both sides of the above equation and using the expression (1.10) for the eigenvalues, one finds (1.2).

Proposition 2. The Bannai-Ito algebra with defining relations (1.5) is isomorphic to the (universal) degenerate double affine Hecke algebra (1.13).

Proof. Let $K_{1}, K_{2}, K_{3}$ satisfy the Bannai-Ito algebra (1.5) with arbitrary structure constants or central elements $\omega_{1}, \omega_{2}, \omega_{3}$ and consider the combinations

$$
\begin{array}{ll}
\widetilde{T}_{0}=\frac{1}{4}\left(K_{1}-K_{2}-K_{3}-\frac{1}{2}\right), & \widetilde{T}_{1}=\frac{1}{4}\left(K_{1}+K_{2}+K_{3}-\frac{1}{2}\right), \\
\widetilde{U}_{0}=\frac{1}{4}\left(-K_{1}-K_{2}+K_{3}-\frac{1}{2}\right), & \widetilde{U}_{1}=\frac{1}{4}\left(-K_{1}+K_{2}-K_{3}-\frac{1}{2}\right) .
\end{array}
$$

It is easily verified that each of $\widetilde{T}_{0}^{2}, \widetilde{T}_{1}^{2}, \widetilde{U}_{0}^{2} \widetilde{U}_{1}^{2}$ is central, i.e that they commute with all generators $\widetilde{T}_{0}, \widetilde{T}_{1}, \widetilde{U}_{0}, \widetilde{U}_{1}$. Indeed, one has

$$
\begin{aligned}
& \widetilde{T}_{0}^{2}=\frac{1}{16}\left(Q+\omega_{1}-\omega_{2}-\omega_{3}+\frac{1}{4}\right)=\widetilde{t}_{0}, \\
& \widetilde{T}_{1}^{2}=\frac{1}{16}\left(Q+\omega_{1}+\omega_{2}+\omega_{3}+\frac{1}{4}\right)=\widetilde{t}_{1}, \\
& \widetilde{U}_{0}^{2}=\frac{1}{16}\left(Q-\omega_{1}-\omega_{2}+\omega_{3}+\frac{1}{4}\right)=\widetilde{u}_{0}, \\
& \widetilde{U}_{1}^{2}=\frac{1}{16}\left(Q-\omega_{1}+\omega_{2}-\omega_{3}+\frac{1}{4}\right)=\widetilde{u}_{1} .
\end{aligned}
$$

Moreover, it is obvious that

$$
\widetilde{T}_{0}+\widetilde{T}_{1}+\widetilde{U}_{0}+\widetilde{U}_{1}=-1 / 2 .
$$

Hence (2.3) provides an algebra map from the Bannai-Ito algebra to the universal degenerate double affine Hecke algebra (1.13). Let us now exhibit the inverse mapping. Let $T_{i}$ and $U_{i}$ for $i=0,1$ satisfy (1.13) and consider the linear combinations

$$
A_{1}=2 T_{0}+2 T_{1}+1 / 2, A_{2}=-2 T_{0}-2 U_{0}-1 / 2, A_{3}=2 T_{1}+2 U_{0}+1 / 2 .
$$


Then by a direct calculation, one finds

$$
\begin{aligned}
& \left\{A_{1}, A_{2}\right\}=A_{3}+4\left(t_{1}^{2}-t_{0}^{2}+u_{0}^{2}-u_{1}^{2}\right), \\
& \left\{A_{2}, A_{3}\right\}=A_{1}+4\left(t_{1}^{2}+t_{0}^{2}-u_{0}^{2}-u_{1}^{2}\right), \\
& \left\{A_{3}, A_{1}\right\}=A_{2}+4\left(t_{1}^{2}-t_{0}^{2}-u_{0}^{2}+u_{1}^{2}\right) .
\end{aligned}
$$

Hence the combinations (2.4) realize the Bannai-Ito relations (1.5). In the realization (2.4), the Casimir operator of the Bannai-Ito algebra has the expression

$$
A_{1}^{2}+A_{2}^{2}+A_{3}^{2}=4\left(t_{0}^{2}+t_{1}^{2}+u_{0}^{2}+u_{1}^{2}\right)-1 / 4 .
$$

Hence the Bannai-Ito algebra (1.5) and the degenerate double affine Hecke algebra (1.13) are isomorphic.

\section{3. "Continuous" Bannai-Ito polynomials AND A NON-COMPACT FORM OF THE BANNAI-ITO ALGEBRA}

In this section, we exhibit a positive-definite orthogonality measure on the real line for the Bannai-Ito polynomials. We relate the existence of this measure to a non-compact form of the BI algebra and present a four-parameter family of irreducible representations stemming from the BI recurrence relation.

Consider the modified Bannai-Ito polynomials, denoted by $\mathbf{Q}_{n}(x ; \mathfrak{a}, \mathfrak{b}, \mathfrak{c}, \mathfrak{d})$, that are obtained by taking

$$
\mathbf{Q}_{n}(x ; \mathfrak{a}, \mathfrak{b}, \mathfrak{c}, \mathfrak{d})=(-i)^{n} \mathbf{B}_{n}(i z ; \mathfrak{a}, \mathfrak{b}, \mathfrak{c}, \mathfrak{d}) .
$$

It follows from (1.1) that the polynomials $\mathbf{Q}_{n}(x)$ satisfy a three-term recurrence relation of the form

$$
x \mathbf{Q}_{n}(x)=\mathbf{Q}_{n+1}(x)+c_{n} \mathbf{Q}_{n}(x)+u_{n} \mathbf{Q}_{n}(x),
$$

where the recurrence coefficients are given by

$$
c_{n}=-i\left(2 \mathfrak{a}+1-A_{n}-C_{n}\right), \quad u_{n}=-A_{n-1} C_{n} .
$$

It is verified that the polynomials $\mathbf{Q}_{n}(x ; \mathfrak{a}, \mathfrak{b}, \mathfrak{c}, \mathfrak{d})$ enjoy the symmetries

$$
\begin{gathered}
\mathbf{Q}_{n}(x ; \mathfrak{a}, \mathfrak{b}, \mathfrak{c}, \mathfrak{d})=\mathbf{Q}_{n}(x ; \mathfrak{b}, \mathfrak{a}, \mathfrak{c}, \mathfrak{d}), \quad \mathbf{Q}_{n}(x ; \mathfrak{a}, \mathfrak{b}, \mathfrak{c}, \mathfrak{d})=\mathbf{Q}_{n}(x ; \mathfrak{a}, \mathfrak{b}, \mathfrak{d}, \mathfrak{c}), \\
\mathbf{Q}_{n}(x ; \mathfrak{a}, \mathfrak{b}, \mathfrak{c}, \mathfrak{d})=(-1)^{n} \mathbf{Q}_{n}(-x ; \mathfrak{c}, \mathfrak{d}, \mathfrak{a}, \mathfrak{b}) .
\end{gathered}
$$

Assume now that the parameters $\mathfrak{a}, \mathfrak{b}, \mathfrak{c}$ and $\mathfrak{d}$ are such that

$$
\overline{\mathfrak{a}}=\mathfrak{c} \text { or } \mathfrak{d}, \quad \overline{\mathfrak{b}}=\mathfrak{d} \text { or } \mathfrak{c},
$$

where $\bar{x}$ stands for complex conjugation. This condition can be implemented, for example, by taking

$$
\mathfrak{a}=\alpha+i \beta, \quad \mathfrak{b}=\gamma+i \delta, \quad \mathfrak{c}=\alpha-i \beta, \quad \mathfrak{d}=\gamma-i \delta,
$$

where $\alpha, \beta, \gamma, \delta$ are real parameters. Under the parametrization (3.4), it is directly verified that the recurrence coefficients $c_{n}$ are of the form

$$
c_{n}= \begin{cases}2 \beta-\frac{(n+4 \alpha+2)(\beta-\delta)}{(n+2 \alpha+2 \gamma+2)}-\frac{n(\beta+\delta)}{(n+2 \alpha+2 \gamma+1)} & n \text { even, } \\ 2 \beta-\frac{(n+4 \alpha+4 \gamma+3)(\beta+\delta)}{n+2 \alpha+2 \gamma+2}-\frac{(n+4 \gamma+1)(\beta-\delta)}{n+2 \alpha+2 \gamma+1} & n \text { odd, }\end{cases}
$$


and hence real for all $n=0,1, \ldots$. The coefficients $u_{n}$ are of the form

$$
u_{n}= \begin{cases}\frac{n(n+4 \alpha+4 \gamma+2)\|n+2[\alpha+\gamma+i(\beta+\delta)]+1\|^{2}}{4(n+2 \alpha+2 \gamma+1)^{2}} & n \text { even, } \\ \frac{(n+4 \alpha+1)(n+4 \gamma+1)\|n+2[\alpha+\gamma+i(\beta-\delta)]+1\|^{2}}{4(n+2 \alpha+2 \gamma+1)^{2}} & n \text { odd. }\end{cases}
$$

It is seen that if $\alpha, \beta, \gamma, \delta$ are positive, then $u_{n}>0$ for all $n=0,1, \ldots$. It hence follows from general theory that the polynomials $\mathbf{Q}_{n}(x)$ form an infinite family of orthogonal polynomials with respect to a positive-definite measure [12.

Proposition 3. Let $\mathfrak{a}$ and $\mathfrak{b}$ be complex numbers with positive real and imaginary parts and let $\mathfrak{c}=\overline{\mathfrak{a}}$ or $\overline{\mathfrak{b}}$ and $\mathfrak{d}=\overline{\mathfrak{b}}$ or $\overline{\mathfrak{a}}$. The Bannai-Ito polynomials $\mathbf{B}_{n}(x ; \mathfrak{a}, \mathfrak{b}, \mathfrak{c}, \mathfrak{d})$ satisfy the orthogonality relation

$$
\frac{1}{4 \pi} \int_{-\infty}^{\infty} W(z) \mathbf{B}_{n}(z) \mathbf{B}_{m}(z) \mathrm{d} z=h_{0} \delta_{n m} \prod_{k=1}^{n} u_{k},
$$

where the positive weight function $W(z)$ is given by

$$
W(z)=\left|\frac{\Gamma(\mathfrak{a}+i z / 2+1) \Gamma(\mathfrak{b}+i z / 2+1) \Gamma(\mathfrak{c}+i z / 2+1 / 2) \Gamma(\mathfrak{d}+i z / 2+1 / 2)}{\Gamma(1 / 2+i z)}\right|^{2},
$$

and where $h_{0}$ reads

$h_{0}=\frac{\Gamma(\mathfrak{a}+\mathfrak{b}+3 / 2) \Gamma(\mathfrak{a}+\mathfrak{c}+1) \Gamma(\mathfrak{b}+\mathfrak{c}+1) \Gamma(\mathfrak{a}+\mathfrak{d}+1) \Gamma(\mathfrak{b}+\mathfrak{d}+1) \Gamma(\mathfrak{c}+\mathfrak{d}+3 / 2)}{\Gamma(\mathfrak{a}+\mathfrak{b}+\mathfrak{c}+\mathfrak{d}+2)}$.

Proof. The orthogonality property follows directly from (1.11) while the normalization coefficients follow from general theory and from comparison with the Wilson integral [14]; see also [10].

From (1.2), it follows that the modified Bannai-Ito polynomials $\mathbf{Q}_{n}(x ; \mathfrak{a}, \mathfrak{b}, \mathfrak{c}, \mathfrak{d})$ defined by (3.1) satisfy the eigenvalue equation

$$
\mathcal{M} \mathbf{Q}_{n}(x)=\lambda_{n} \mathbf{Q}_{n}(x), \quad \lambda_{n}=(-1)^{n}(n+\mathfrak{a}+\mathfrak{b}+\mathfrak{c}+\mathfrak{d}+3 / 2),
$$

where $\mathcal{M}$ is given by

$$
\begin{aligned}
\mathcal{M} & =\left(\frac{(2 \mathfrak{a}+1-i x)(2 \mathfrak{b}+1-i x)}{1-2 i x}\right)\left(S_{x}^{+} R_{x}-\mathbb{1}\right) \\
& +\left(\frac{(2 \mathfrak{c}+1+i x)(2 \mathfrak{d}+1+i x)}{1+2 i x}\right)\left(S_{x}^{-} R_{x}-\mathbb{1}\right)+(\mathfrak{a}+\mathfrak{b}+\mathfrak{c}+\mathfrak{d}+3 / 2) \mathbb{1},
\end{aligned}
$$

where $S_{x}^{ \pm} f(x)=f(x \pm i)$ are the imaginary shift operators. It is manifest that under the conditions (3.3), the eigenvalues $\lambda_{n}$ in (3.8) are real and the operator $\mathcal{M}$ is self-adjoint. The operators $\mathcal{M}$ and $X$ generate a non-compact form of the Bannai-Ito algebra. Indeed, upon defining

$$
A_{1}=\mathcal{M}, \quad A_{2}=X,
$$

a direct calculation shows that one has

$$
\left\{A_{1}, A_{2}\right\}=A_{3}+\alpha_{3}, \quad\left\{A_{2}, A_{3}\right\}=-A_{1}+\alpha_{1}, \quad\left\{A_{3}, A_{1}\right\}=A_{2}+\alpha_{2},
$$


where the first relation of (3.11) is taken to define $A_{3}$ and where the structure constants read

$$
\begin{aligned}
& \alpha_{1}=-[4(\mathfrak{a} \mathfrak{b}+\mathfrak{c} \mathfrak{d})+\mathfrak{a}+\mathfrak{b}+\mathfrak{c}+\mathfrak{d}+1 / 2], \\
& \alpha_{2}=-i\left[2\left(\mathfrak{a}^{2}+\mathfrak{b}^{2}-\mathfrak{c}^{2}-\mathfrak{d}^{2}\right)+\mathfrak{a}+\mathfrak{b}-\mathfrak{c}-\mathfrak{d}\right], \\
& \alpha_{3}=-i[4(\mathfrak{a} \mathfrak{b}-\mathfrak{c} \mathfrak{d})+\mathfrak{a}+\mathfrak{b}-\mathfrak{c}-\mathfrak{d}] .
\end{aligned}
$$

When $\mathfrak{c}=\overline{\mathfrak{a}}$ or $\overline{\mathfrak{b}}$ and $\mathfrak{d}=\overline{\mathfrak{b}}$ or $\overline{\mathfrak{a}}$, the structure constants (3.12) are real. The algebra (3.11) differs from the standard Bannai-Ito algebra (1.5) by a change of sign in the anticommutation relations and can thus be viewed as a non-compact form of the latter. The difference between (1.5) and (3.11) is similar in spirit to the difference that exists between $\mathfrak{s u}(2)$ and $\mathfrak{s u}(1,1)$. The Casimir operator $Z$ for the algebra (3.11) is naturally given by

$$
Z=A_{1}^{2}-A_{2}^{2}-A_{3}^{2} \text {. }
$$

In the realization (3.10), it takes the same value as in (1.7).

In light of the eigenvalue equation (3.8) and the recurrence relation (3.2), one can straightforwardly introduce a four-parameter family of infinite-dimensional, self-adjoint representations of the algebra (3.11). This is done in the following proposition.

Proposition 4. Let $\alpha, \beta, \gamma, \delta$ be positive real numbers and consider the infinitedimensional vector space $V$ spanned by the basis vectors $e_{n}, n=0,1, \ldots$, endowed with the actions

$$
\begin{aligned}
& A_{1} e_{n}=(-1)^{n}(n+2 \alpha+2 \gamma+3 / 2) e_{n}, \\
& A_{2} e_{n}=\sqrt{u_{n+1}} e_{n+1}+c_{n} e_{n}+\sqrt{u_{n}} e_{n-1},
\end{aligned}
$$

where $u_{n}$ and $c_{n}$ are given by (3.5) and (3.6), respectively. Then $V$ supports an infinite-dimensional, irreducible, self-adjoint representation of the non-compact Bannai-Ito algebra (3.11).

Proof. One can verify by direct calculation that with the actions (3.13), the generators $A_{1}, A_{2}$ satisfy the relations (3.11). The fact that the representation is self-adjoint follows from the observation that for positive values of $\alpha, \beta, \gamma, \delta$, the coefficients $c_{n}$ are real and the coefficients $u_{n}$ are positive for all $n=0,1, \ldots$. The irreducibility is a consequence of the fact that under these conditions, $u_{n} \neq 0$ for all non-negative integers $n$.

\section{Conclusion}

In this paper, we have established the relationship between the Bannai-Ito polynomials and the non-symmetric Wilson polynomials as well as between the BannaiIto algebra and a rank-one degenerate double affine Hecke algebra associated with the root system $\left(C_{1}^{\vee}, C_{1}\right)$. We have also exhibited a positive-definite, continuous measure for the BI polynomials, displayed the associated non-compact form of the BI algebra and presented a family of irreducible representations.

The results presented suggest several questions of interest. First, given the applications of the one-variable Bannai-Ito polynomials to exactly solvable models (e.g. [4,8]), it would be of interest to study the multivariate non-symmetric Wilson polynomials introduced in 11 from that perspective; the results of such an investigation should be compared for example with those found in [26] and [27]. Second, in view 
of the fact that the Bannai-Ito polynomials $\mathbf{B}_{n}(x)$ obeying a discrete and finite orthogonality relation arise as Racah coefficients for positive-discrete series representations of $\mathfrak{o s p}(1 \mid 2)$ [], it would be natural to look for a similar interpretation for the modified Bannai-Ito polynomials $\mathbf{Q}_{n}(x)$, which satisfy a continuous orthogonality relation. This would possibly involve different types of representations other than those of the positive-discrete series; see 9

\section{ACKNowledGments}

The authors would like to thank S. Tsujimoto and W. Groenevelt for discussions. The first author was supported by the Natural Science and Engineering Research Council of Canada (NSERC). The research of the second author was supported in part by NSERC. The third author would like to thank the Centre de recherches mathématiques (CRM) for its hospitality.

\section{REFERENCES}

[1] George E. Andrews, Richard Askey, and Ranjan Roy, Special functions, Encyclopedia of Mathematics and its Applications, vol. 71, Cambridge University Press, Cambridge, 1999. MR.1688958

[2] Eiichi Bannai, Orthogonal polynomials in coding theory and algebraic combinatorics, Orthogonal polynomials (Columbus, OH, 1989), NATO Adv. Sci. Inst. Ser. C Math. Phys. Sci., vol. 294, Kluwer Acad. Publ., Dordrecht, 1990, pp. 25-53, DOI 10.1007/978-94-009-0501-6_2. MR.1100287

[3] Eiichi Bannai and Tatsuro Ito, Algebraic combinatorics. I, Association schemes. The Benjamin/Cummings Publishing Co., Inc., Menlo Park, CA, 1984. MR882540

[4] Vincent X. Genest, Luc Vinet, and Alexei Zhedanov, A Laplace-Dunkl equation on $S^{2}$ and the Bannai-Ito algebra, Comm. Math. Phys. 336 (2015), no. 1, 243-259, DOI 10.1007/s00220014-2241-4. MR.3322373

[5] Pavel Etingof, Alexei Oblomkov, and Eric Rains, Generalized double affine Hecke algebras of rank 1 and quantized del Pezzo surfaces, Adv. Math. 212 (2007), no. 2, 749-796, DOI 10.1016/j.aim.2006.11.008. MR.2329319

[6] Vincent X. Genest, Luc Vinet, and Alexei Zhedanov, Bispectrality of the complementary Bannai-Ito polynomials, SIGMA Symmetry Integrability Geom. Methods Appl. 9 (2013), Paper 018, 20, DOI 10.3842/SIGMA.2013.018. MR3033560

[7] Vincent X. Genest, Luc Vinet, and Alexei Zhedanov, The Bannai-Ito polynomials as Racah coefficients of the $s_{-1}(2)$ algebra, Proc. Amer. Math. Soc. 142 (2014), no. 5, 1545-1560, DOI 10.1090/S0002-9939-2014-11970-8. MR3168462

[8] Vincent X. Genest, Luc Vinet, and Alexei Zhedanov, A Laplace-Dunkl equation on $S^{2}$ and the Bannai-Ito algebra, Comm. Math. Phys. 336 (2015), no. 1, 243-259, DOI 10.1007/s00220014-2241-4. MR.3322373

[9] Wolter Groenevelt, Wilson function transforms related to Racah coefficients, Acta Appl. Math. 91 (2006), no. 2, 133-191, DOI 10.1007/s10440-006-9024-7. MR2249545

[10] Wolter Groenevelt, Fourier transforms related to a root system of rank 1, Transform. Groups 12 (2007), no. 1, 77-116, DOI 10.1007/s00031-005-1124-5. MR2308030

[11] Wolter Groenevelt, Multivariable Wilson polynomials and degenerate Hecke algebras, Selecta Math. (N.S.) 15 (2009), no. 3, 377-418, DOI 10.1007/s00029-009-0005-3. MR2551187

[12] Mourad E. H. Ismail, Classical and quantum orthogonal polynomials in one variable, with two chapters by Walter Van Assche; with a foreword by Richard A. Askey; reprint of the 2005 original. Encyclopedia of Mathematics and its Applications, vol. 98, Cambridge University Press, Cambridge, 2009. MR.2542683

[13] M. E. H. Ismail, E. Rains, and D. Stanton, Orthogonality of very well-poised series, 2015.

[14] Roelof Koekoek, Peter A. Lesky, and René F. Swarttouw, Hypergeometric orthogonal polynomials and their q-analogues, Springer Monographs in Mathematics, Springer-Verlag, Berlin, 2010. With a foreword by Tom H. Koornwinder. MR2656096 
[15] Tom H. Koornwinder, Askey-Wilson polynomials for root systems of type BC, Hypergeometric functions on domains of positivity, Jack polynomials, and applications (Tampa, FL, 1991), Contemp. Math., vol. 138, Amer. Math. Soc., Providence, RI, 1992, pp. 189-204, DOI 10.1090/conm/138/1199128. MR/199128

[16] Tom H. Koornwinder, The relationship between Zhedanov's algebra AW(3) and the double affine Hecke algebra in the rank one case, SIGMA Symmetry Integrability Geom. Methods Appl. 3 (2007), Paper 063, 15, DOI 10.3842/SIGMA.2007.063. MR2299864

[17] Tom H. Koornwinder, Zhedanov's algebra AW(3) and the double affine Hecke algebra in the rank one case. II. The spherical subalgebra, SIGMA Symmetry Integrability Geom. Methods Appl. 4 (2008), Paper 052, 17, DOI 10.3842/SIGMA.2008.052. MR2425640

[18] Douglas A. Leonard, Orthogonal polynomials, duality and association schemes, SIAM J. Math. Anal. 13 (1982), no. 4, 656-663, DOI 10.1137/0513044. MR661597

[19] I. G. Macdonald, Symmetric functions and orthogonal polynomials, Dean Jacqueline B. Lewis Memorial Lectures presented at Rutgers University, New Brunswick, NJ, University Lecture Series, vol. 12, American Mathematical Society, Providence, RI, 1998. MR1488699

[20] S. Shahi, Non-symmetric Koornwinder polynomials and duality, Annals of Mathematics, 150:267-282, 1999.

[21] Jasper V. Stokman, Koornwinder polynomials and affine Hecke algebras, Internat. Math. Res. Notices 19 (2000), 1005-1042, DOI 10.1155/S1073792800000520. MR 1792347

[22] Paul Terwilliger, The universal Askey-Wilson algebra and DAHA of type $\left(C_{1}^{\vee}, C_{1}\right)$, SIGMA Symmetry Integrability Geom. Methods Appl. 9 (2013), Paper 047, 40, DOI 10.3842/SIGMA.2013.047. MR3116183

[23] Satoshi Tsujimoto, Luc Vinet, and Alexei Zhedanov, Dunkl shift operators and BannaiIto polynomials, Adv. Math. 229 (2012), no. 4, 2123-2158, DOI 10.1016/j.aim.2011.12.020. MR2880217

[24] Satoshi Tsujimoto, Luc Vinet, and Alexei Zhedanov, Dual -1 Hahn polynomials: "classical" polynomials beyond the Leonard duality, Proc. Amer. Math. Soc. 141 (2013), no. 3, 959-970, DOI 10.1090/S0002-9939-2012-11469-8. MR3003688

[25] J. F. van Diejen, Commuting difference operators with polynomial eigenfunctions, Compositio Math. 95 (1995), no. 2, 183-233. MR.1313873

[26] J. F. van Diejen, Multivariable continuous Hahn and Wilson polynomials related to integrable difference systems, J. Phys. A 28 (1995), no. 13, L369-L374. MR1352361

[27] J. F. van Diejen and E. Emsiz, Discrete harmonic analysis on a Weyl alcove, J. Funct. Anal. 265 (2013), no. 9, 1981-2038, DOI 10.1016/j.jfa.2013.06.023. MR3084495

[28] L. Vinet and J. F. Van Diejen, editors. Calogero-Moser-Sutherland models. Springer, 2000. MR.1843558

[29] Luc Vinet and Alexei Zhedanov, A limit $q=-1$ for the big $q$-Jacobi polynomials, Trans. Amer. Math. Soc. 364 (2012), no. 10, 5491-5507, DOI 10.1090/S0002-9947-2012-05539-5. MR2931336

[30] A. S. Zhedanov, "Hidden symmetry" of Askey-Wilson polynomials (Russian, with English summary), Teoret. Mat. Fiz. 89 (1991), no. 2, 190-204, DOI 10.1007/BF01015906; English transl., Theoret. and Math. Phys. 89 (1991), no. 2, 1146-1157 (1992). MR.1151381

Department of Mathematics, Massachusetts Institute of Technology, Cambridge, MASSACHUSETTS 02139

E-mail address: vxgenest@mit.edu

Centre de recherches mathématiques, Université de Montréal, Montréal, QC, Canada H3C 3J7

E-mail address: vinetl@crm.umontreal.ca

Donetsk Institute for Physics And Technology, Donetsk 83114, Ukraine

E-mail address: zhedanov@yahoo.com 